University of Nebraska - Lincoln

DigitalCommons@University of Nebraska - Lincoln

Rerouting Schemes for Dynamic Traffic Grooming in Optical WDM Mesh Networks

\author{
Wang Yao \\ University of Nebraska-Lincoln, wyao@cse.unl.edu \\ Byrav Ramamurthy \\ University of Nebraska-Lincoln, bramamurthy2@unl.edu
}

Follow this and additional works at: https://digitalcommons.unl.edu/cseconfwork

Part of the Computer Sciences Commons

Yao, Wang and Ramamurthy, Byrav, "Rerouting Schemes for Dynamic Traffic Grooming in Optical WDM Mesh Networks" (2004). CSE Conference and Workshop Papers. 113.

https://digitalcommons.unl.edu/cseconfwork/113

This Article is brought to you for free and open access by the Computer Science and Engineering, Department of at DigitalCommons@University of Nebraska - Lincoln. It has been accepted for inclusion in CSE Conference and Workshop Papers by an authorized administrator of DigitalCommons@University of Nebraska - Lincoln. 


\title{
Rerouting Schemes for Dynamic Traffic Grooming in Optical WDM Mesh Networks
}

\author{
Wang Yao and Byrav Ramamurthy \\ Department of Computer Science and Engineering, \\ University of Nebraska-Lincoln, Lincoln NE 68588 USA \\ Email: \{wyao,byrav\}@cse.unl.edu
}

\begin{abstract}
Traffic grooming in optical WDM mesh networks is a two-layer routing problem to effectively pack low-rate connections onto high-rate lightpaths, which, in turn, are established on wavelength links. In this work, we employ the rerouting approach to improve the network throughput under the dynamic traffic model. We propose two rerouting schemes, rerouting at lightpath level (RRAL) and rerouting at connection level (RRAC). A qualitative comparison is made between RRAL and RRAC. We also propose the critical-wavelength-avoiding one-lightpath-limited (CWA-1L) and critical-lightpath-avoiding one-connection-limited (CLA-1C) rerouting heuristics, which are based on the two rerouting schemes respectively. Simulation results show that rerouting reduces the connection blocking probability significantly.
\end{abstract}

\section{INTRODUCTION}

Wavelength division multiplexing (WDM) based optical networking architectures using optical cross-connects (OXCs) are promising solutions to the next-generation long-haul transport networks [1]. The transmission rate on a wavelength channel has reached OC-192 (10Gbps) and is expected to reach OC-768 (40Gbps) in the future. While WDM technology enables huge amount of bandwidth, the traffic demand is increasing at an explosive rate as well. The traffic demand granularity varies a lot, possibly from OC-3 (155Mbps) to OC192 (10Gbps). The routing problem with the bandwidth gap between the low-rate connection and high-rate wavelength channels is addressed as a two-layer traffic grooming problem [2]-[3] with the goal to effectively share resources in the optical networks.

The two layers involved in traffic grooming are optical layer and electronic layer. The optical layer is composed of OXCs with point-to-point fiber links connecting them. The OXC nodes and the fiber links constitute the physical topology of an optical network. The optical layer establishes lightpaths along wavelength channels within the fibers. All the lightpaths and their corresponding end nodes constitute the virtual (logical) topology. The electronic layer establishes traffic connections on top of the virtual topology.

Optimal routing in a two-layered optical network with dynamically arriving connection requests is a challenging task. Various factors may deteriorate the route selection of lightpaths and connections and thus reduce the total network throughput. For example, the resources contention between two lightpaths (or connections) may result in the blocking of one lightpath or the use of an inefficient long path for one of the two lightpaths. In a dynamic traffic environment, the contention is more prominent a problem, as the arrivals and departures of connections are not known in advance.
Therefore, it is not possible to make the optimal routing decisions for all the lightpaths and connections at the same time so that the total network throughput can be maximized. As another example, consider optical networks without wavelength conversion capability. The wavelength continuity constraint stipulates that a lightpath must utilize the same wavelength along its path. This constraint reduces the possibility of successfully finding a free wavelength on a path and thus may force the lightpath to use another longer path or get blocked.

Rerouting is a useful technique to address the routing challenges mentioned above. While wavelength conversion is one technique to alleviate the inefficiency caused by wavelength continuity constraints, the work in [4] shows that rerouting also helps to increase the overall resource utilization efficiency. In [4], a move-to-vacant wavelength-retuning (MTV_WR) rerouting scheme was proposed. MTV means rerouting the lightpath to a path along which at least one wavelength is not occupied by any other lightpath. Using MTV scheme, a new lightpath could be established on the new path before stopping the transmission on the old path and switching to the new path. Therefore, MTV achieves a small rerouting disruption time. On the other hand, WR means only retuning the wavelength of the lightpath on the same path. It makes the rerouting algorithm and operation simple. By combining the two schemes, MTV_WR tries to retune a lightpath to a free wavelength on the same path. It has the advantages of both MTV and WR. However, WR is not able to reduce the resource-inefficiency caused by lightpaths using extremely long paths. In [4], MTV_WR was used in the second phase of an overall two-phase routing scheme. The first phase is a normal routing process without considering rerouting. If the first phase fails to find a path for the lightpath request, then the MTV_WR rerouting is applied. In [5], a single-phase algorithm still using MTV_WR scheme but combining routing and rerouting together was presented. It claims to have less time complexity than the two-phase algorithm.

In this paper, we study the rerouting approach for the provisioning of multi-granularity connections in two-layer wavelength-routed optical networks with grooming capability. We consider the dynamic traffic environment where connection requests arrive and depart dynamically. As traffic grooming aims at improving the network resource utilization efficiency, we expect that using rerouting in traffic grooming will help achieve the objective. As in [4], we use a two-phase routing scheme. The rerouting procedure is applied only when the normal routing fails. We propose two rerouting schemes at 
two different levels, namely rerouting at lightpath level (RRAL) and rerouting at connection level (RRAC). To fully exploit the ability of rerouting to reduce the long path inefficiency, we use the idea of MTV, instead of MTV_WR, in the RRAL and RRAC rerouting schemes. We show that both schemes still preserve short disruption time property while reducing the blocking probability of connection provisioning. Two efficient heuristic rerouting algorithms based on RRAL and RRAC are proposed as well.

The rest of the paper is organized as follows: Section II presents the two rerouting schemes. Section III presents two heuristic rerouting algorithms. Section IV discusses the simulation results.

\section{REROUTING SCHEMES}

A complete rerouting scheme generally has two components: rerouting algorithm and rerouting operation [4][5]. A rerouting algorithm determines whether in order to accommodate a new connection, existing lightpaths or connections will be rerouted, and if they are rerouted, which new paths they will use. The rerouting operation decides the sequence of steps executed in the networks to migrate the rerouted lightpaths or connections to their new paths. The rerouting operation belongs to the function of control plane and largely determines the rerouting disruption time. For highspeed optical networks, even a short disruption time may affect a large amount of traffic. Therefore, it is desirable that a rerouting scheme should incur the minimum disruption time.

As the rerouting operation belongs to the functionality of the control plane, it can be implemented using generalized multiprotocol label switching (GMPLS) [6], which is expected to be the unified control plane for next-generation optical networks. GMPLS supports multiple switching types such as timedivision multiplexing (TDM), wavelength (lambda) and fiber (port) switching. Accordingly, GMPLS supports a hierarchy of label switched paths (LSPs), which correspond to channels such as lightpaths at the wavelength level and SONET circuits at the subwavelength level. In this section, we propose two rerouting schemes, RRAL and RRAC.

\section{A. Rerouting at Lightpath (RRAL) Level}

\section{1) Rerouting algorithm}

The basic idea of RRAL is to reroute some of the existing lightpaths so that new lightpaths can be established to carry an otherwise blocked connection. For example, for a network in Fig. 1 (a), assume that each fiber has only one wavelength $\lambda_{0}$ and a wavelength can carry two connections. Fig. 1 (b) shows the current virtual topology and connections carried over the virtual topology. If a connection request $0-1$ arrives, it cannot be accepted because neither does an existing lightpath $0-1$ exist nor a new lightpath $0-1$ can be established. The wavelength $\lambda_{0}$ on link $0-1$ has been allocated to the lightpath $0-4$ which uses path 0-1-4. However, if the lightpath $0-4$ is rerouted to path 0-3-4, as shown in Fig. 1 (d), then a new lightpath $0-1$ can be established to carry the new connection 0 1. While the new lightpath established after rerouting can be the only lightpath needed to carry the connection, it is also

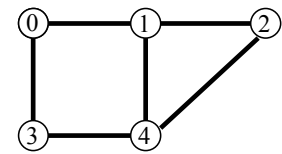

(a)

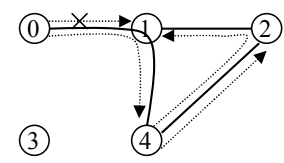

(c)

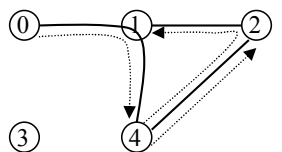

(b)

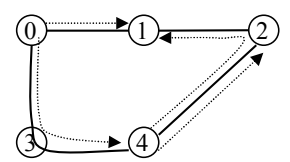

(d) $\longrightarrow$ Fiber $\longrightarrow$ Lightpath $\quad \rightarrow$ Connection

Fig. 1. Illustration of rerouting at lightpath level. Assume that each fiber has one wavelength $\lambda_{0}$ and each wavelength can accommodate two connections. (a) Physical topology, (b) Virtual topology and connections, (c) Connection 01 denied before rerouting, (d) Connection 0-1 accepted after rerouting.

possible that the new lightpath is one hop of the path to carry the connection.

\section{2) Rerouting operation}

The lightpath rerouting operation is executed when the rerouting algorithm has decided which lightpaths are to be rerouted and their new paths. As multiple lightpaths may be rerouted, we assume that they are computed by the rerouting algorithm in such a way that they can be rerouted in parallel. This parallelism saves the time needed to wait for the completion of the rerouting process. The following procedure describes the basic steps to execute a lightpath rerouting operation. Once the rerouting operation finishes, the routing protocol can begin to establish the new connection.

For each lightpath to be rerouted:

1) Establish a new LSP for the rerouted lightpath.

2) Stop the data transmission on all connections using the rerouted lightpath.

3) Switch the rerouted lightpath from its old LSP to the new LSP.

4) Start the data transmission on all connections involved.

5) Release the wavelengths and transceivers used by the rerouted lightpath on the old LSP.

\section{B. Rerouting at Connection (RRAC) Level}

1) Rerouting algorithm

The basic idea of RRAC is to reroute some existing connections from certain lightpaths so that the otherwise blocked connection can use these lightpaths in its path. For example, for the network in Fig. 2 (a), assume that Fig. 2 (b) shows its current virtual topology and connections. If a connection request 3-2 arrives, it will be blocked, because all the bandwidth capacity on lightpath 3-4 has been used by two other connections. However, if the connection $0-4$ can be rerouted to lightpath $0-4$ which uses path $0-1-4$, as shown in Fig. 2 (d), then the connection 3-2 can be carried over a twohop (virtual) path 3-4-2 using lightpaths 3-4 and 4-2.

\section{2) Rerouting operation}

The connection rerouting operation is executed when the rerouting algorithm has determined which connections are to be rerouted and their new paths. Note that in order to establish the new path for a rerouted connection, new lightpaths may need to be established. 


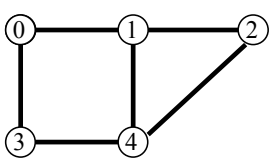

(a)

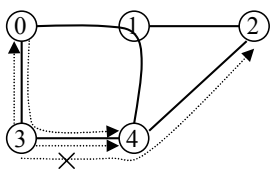

(c)

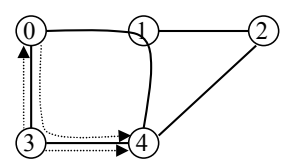

(b)

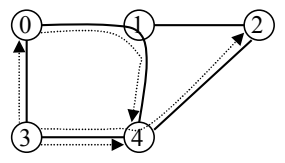

(d) $\longrightarrow$ Fiber $\longrightarrow$ Lightpath $\quad \rightarrow$ Connection

Fig. 2. Illustration of rerouting at connection level. Assume that each fiber has one wavelength $\lambda_{0}$ and each wavelength can accommodate two connections. (a) Physical topology, (b) Virtual topology and connections, (c) Connection 3-2 denied before rerouting, (d) Connection 3-2 accepted after rerouting.

For each connection to be rerouted:

1) Establish a new LSP for the rerouted connection. Establish new lightpaths if necessary.

2) Stop the data transmission on the rerouted connection.

3) Switch the rerouted connection from its old LSP to the new LSP.

4) Start the data transmission on the rerouted connection.

5) Release the lightpath bandwidth allocated in the old LSP of the rerouted connection.

\section{Comparison of RRAL and RRAC}

Generally, RRAL is a coarse-granularity rerouting scheme which operates at the high-rate lightpath (aggregate) level, while RRAC is a fine-granularity rerouting scheme which operates at the low-rate connection (per-flow) level. We compare RRAL and RRAC with respect to rerouting algorithm and rerouting operation respectively.

As a coarse-granularity scheme, the RRAL routing algorithm is relatively simple. It only needs the global information of the lightpaths in the network to decide the rerouted lightpaths and their new paths. The route changes of the underlying lightpaths can be viewed as being transparent to the upper connections. RRAC routing algorithm, on the other hand, needs the global information of all the lightpaths and connections to compute the rerouted connections and their new paths. As the grooming factor $C$ (which is equal to the capacity of a lightpath divided by the smallest allowed connection capacity) is usually a large number, the number of connections in the network is larger than the number of lightpaths. Therefore, the RRAC routing algorithm usually has a larger complexity. However, as a fine-granularity scheme, RRAC is more flexible in terms of selecting rerouted connections and their new paths. Also, as an end-to-end rerouting scheme with respect to connections, RRAC routing algorithm may be good at preserving other quality of service (QoS) and traffic engineering (TE) constraints imposed on the connections.

From the operational point of view, RRAL usually affects more traffic than RRAC during the rerouting process. This is because that in the LSP switch step, RRAL stops the data transmission on all the connections that use the rerouted lightpath, while RRAC only stops the data transmission on the rerouted connection. The service disruption time is low for both RRAL and RRAC. Theoretically, the service disruption time is equal to the time needed to switch from the old LSP to the new LSP. It is very short since the new LSP has been established before disrupting the data transmission.

\section{HeURISTIC REROUTING AlgORITHMS}

Our proposed routing scheme for traffic grooming includes two phases: a normal routing phase and a rerouting phase. Note that when we say routing in this paper, it refers to a general concept which includes the grooming function. The rerouting phase is only executed when the normal routing phase fails to find a path for the arriving connection request. The rerouting phase tries to reroute some lightpaths or connections so that the connection request can be accepted. At the end of the rerouting phase, if those lightpaths or connections are rerouted successfully, then the connection request is provisioned again using the routing algorithm of the normal routing phase. We represent a connection request as $\Phi\left(s, d, t, \Delta_{\mathrm{t}}, B\right)$, where $s$ is source node, $d$ is destination node, $t$ is the arrival time of the traffic request, $\Delta_{t}$ is the required service time for this request and $B$ is the traffic bandwidth requirement.

In this work, the normal routing phase uses the least virtual hop first (LVHF) routing algorithm [7]. LVHF tries to use the least number of virtual hops to carry a connection on the fixed alternate paths. Basically, LVHF has two steps. The first step is to select a physical path in the physical topology as a candidate path for the arriving connection request. Then the second step is to use existing lightpaths or establish new lightpaths on the candidate path to carry the connection. Depending on whether new lightpaths need to be established and the number of lightpaths used, the connection path can be divided into four categories. Note that a (virtual) hop in a connection path is a lightpath in the virtual topology.

- Single-hop path using a new lightpath (SN-path).

- Single-hop path using existing lightpath (SE-path).

- Multi-hop path only using existing lightpaths (MOE-path).

- Multi-hop path using both existing and new lightpaths (MNE-path).

As for the rerouting phase, we propose two rerouting heuristics, namely critical-wavelength-avoiding one-lightpathlimited (CWA-1L) heuristic and critical-lightpath-avoiding one-connection-limited (CLA-1C) heuristic, which are based on RRAL and RRAC respectively. As indicated by their names, CWA-1L and CLA-1C restrict the rerouting operation to one lightpath or connection each time for a connection request. This restriction not only reduces the complexity of the rerouting algorithms, but also reduces the amount of traffic affected by the rerouting operation.

\section{A. CWA-1L Rerouting Heuristic}

Define the critical wavelength set of a path $p C W(p)=$ $\left\{\lambda_{e}: e \in p\right.$ and $\exists l \in L P \lambda_{e} \in l$ and $\left.\forall e^{\prime} \in p-e \forall l \in L P \lambda_{e^{\prime}} \notin l\right\} \quad$, where $\lambda_{e}$ is the wavelength $\lambda$ on the fiber link $e, L P$ is the set of all the lightpaths in the network, $\lambda_{e} \in l$ means that lightpath $l$ uses the wavelength $\lambda_{e}$. With the definition of $C W(p)$, we 
can see that once a $\lambda_{e} \in C W(p)$ becomes free, a new lightpath can be established on $p$ using wavelength $\lambda$. Since any $\lambda_{e} \in C W(p)$ is used by a lightpath, we have to use the rerouting technique to make $\lambda_{e}$ free. Define the lightpath using a critical wavelength $\lambda_{e}$ as the critical wavelength related lightpath (CWR-lightpath). If we can reroute a CWR-lightpath of path $p$ without using the critical wavelength $\lambda_{e}$, then a new lightpath can be established on path $p$ to satisfy the new connection. Fig. 3 shows the CWA-1L heuristic algorithm and the NEW_LP_CWA procedure used in CWA-1L.
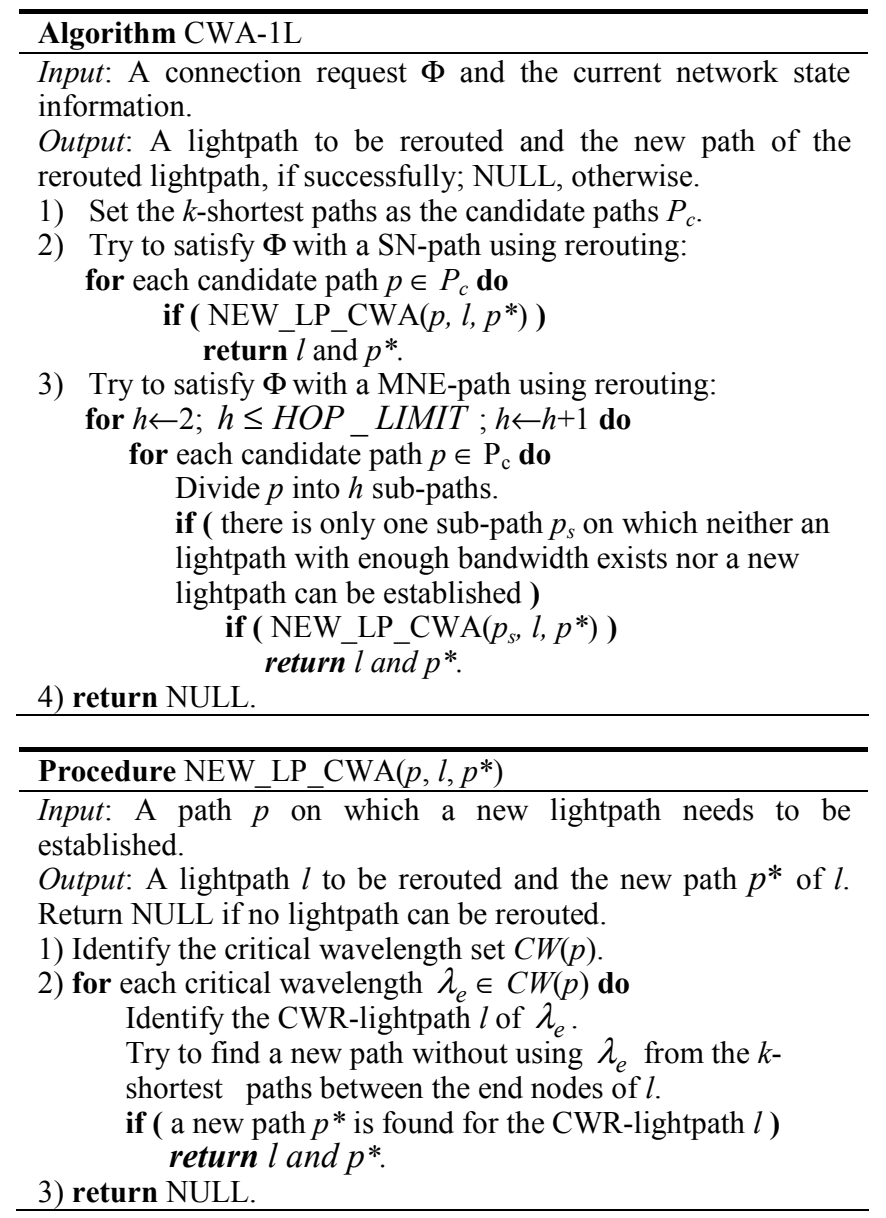

Fig. 3. The CWA-1L algorithm and the NEW_LP_CWA method.

\section{B. CLA-1C Rerouting Heuristic}

Define the critical lightpath set of a path $p$ and a connection request $\Phi C L(p, \Phi)=\{l \in L P: l$ is on path $p\} \cap$

$\left\{l \in L P: B_{a}(l)<B_{\Phi}\right.$ and $\left.\exists c \in C O N(l) B_{c}+B_{a}(l) \geq B_{\Phi}\right\}$,

where $B_{a}(l)$ is the available bandwidth on lightpath $l, B_{\Phi}$ is the bandwidth requirement of connection request $\Phi$, $C O N(l)$ is the set of connections that use lightpath $l, B_{c}$ is the bandwidth of connection $c$. Correspondingly, $c$ is a critical lightpath related connection (CLR-connection) of the path $p$ and request $\Phi$. For a critical lightpath $l$ of $p$ and $\Phi$, all its CLR-connections constitute the CLR-connection set CLRC $(p$, $\Phi, l)$. From the above definition, we can see that if any CLR- connection is rerouted without using the corresponding critical lightpath $l$, then $l$ will have enough free bandwidth capacity to carry the new connection request $\Phi$. Fig. 4 shows the CLA$1 \mathrm{C}$ heuristic algorithm and the FREE_CON_CLA procedure used in CLA-1C.
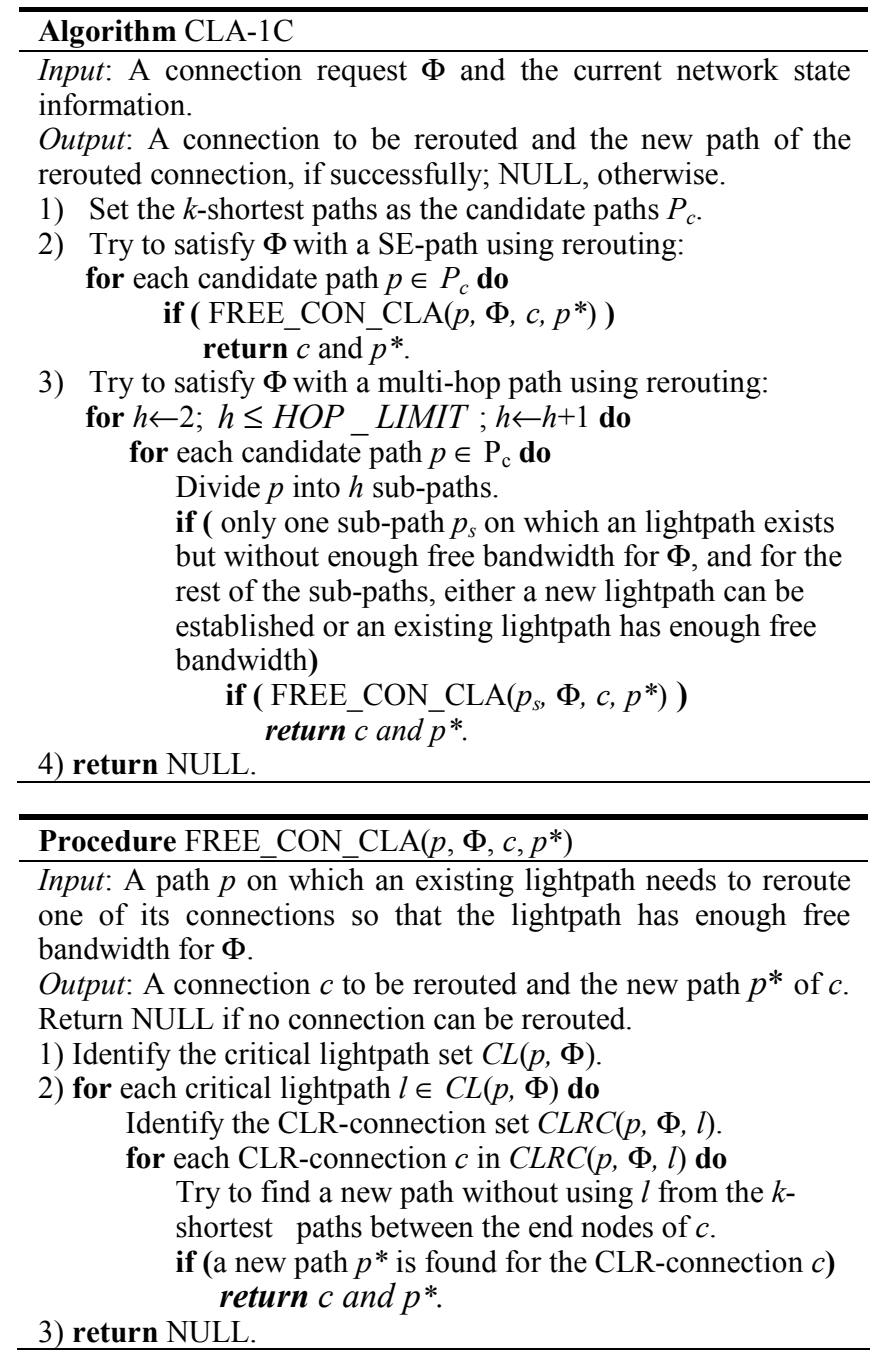

Fig. 4. The CLA-1C algorithm and the FREE_CON_CLA method.

\section{NUMERICAL RESUltS AND DISCUSSION}

To evaluate the performance of the two proposed rerouting heuristics, we simulate them on the NSFNET (14-node 42link) and EUPAN (22-node and 90-link) networks. The following assumptions are used. Connection requests arrive in a Poisson process with rate $\lambda$ and are uniformly distributed among all the node pairs. The connection service time is distributed exponentially with mean $1 / \mu$. Suppose the capacity (C) of a lightpath is normalized by dividing it by the smallest grooming granularity. We assume $C$ is 16 . The connection bandwidth requirement is uniformly distributed between 1 and $C / 2$. Each fiber supports 16 wavelengths and each node in the network has 32 transceivers. In this section, LVHF donates the LVHF routing algorithm without the rerouting phase. CWA$1 \mathrm{~L}$ and CLA-1C donate using LVHF in the normal routing phase and using the CWA-1L and CLA-1C algorithms 
respectively in the rerouting phase.

Fig. 5 compares the performance of CWA-1L and CLA-1C with LVHF on the NSFNET network in terms of connection request blocking probability. From Fig. 5, we can see that with the same $k$ (the number of alternate paths), CWA-IL and CLA$1 \mathrm{C}$ always yield a lower blocking probability than LVHF. The only exception is when $k=1$, CWA-1L does not improve much over LVHF. This is because when we restrict the candidate path to be the shortest path, not many choices are left for CWA-1L to reroute a lightpath. The only possibility is to switch to a different wavelength on the same path. However, this effort seems to be futile according to the simulation results. CLA-1C, on the other hand, performs better than LVHF when $k=1$. This is because that even though the physical path is restricted to the shortest path, CLA-1C can still use different kinds of concatenations of lightpaths to reroute a connection. We can also see that CLA-1C performs better than CWA-1L on the NSFNET network when $k$ is 2 and 4.

Fig. 6 shows the simulation results on the EUPAN network. As can be seen, CWA-1L and CLA-1C perform better than LVHF when $k$ is 2 and 4 . This result, along with the result from Fig. 5, substantiates our claim that rerouting helps to increase the network throughput, and thus enhance the resource utilization. Comparing CWA-1L and CLA-1C, we can see that CWA-1L yields lower blocking probability on the EUPAN network than CLA-1C when $k$ is 2 . When $k$ is 4 , CWA-1L only performs better than CLA-1C under extremely light traffic load and is outperformed by CLA-1C under relatively medium or heavy traffic load. The reason why CWA-1L performs relatively worse than CLA-1C under heavy load is that almost all wavelengths are consumed by other lightpaths in such cases. CWA-1L can hardly find available resources to reroute a lightpath. As for CLA-1C, it is more flexible in utilizing the available bandwidth in the lightpaths to reroute a relatively low-rate connection. Also, the reason why CWA-1L performs relatively worse at large $k$ is that a large $k$ may result in more inefficient long paths being used by lightpaths. Even though it may result in accepting a few specific connections, overall it reduces the resource efficiency. This problem does not exist with CLA-1C because CLA-1C operates at much lower granularities than CWA-1L. A small proportion of the connections using relatively long paths do not significantly reduce the resource efficiency. On the contrary, it reduces the blocking probability by accepting the otherwise rejected connection requests.

By comparing Fig. 5 and Fig. 6, we can see that CWA-1L performs better in a relatively dense network like EUPAN, rather than in a relatively sparse network like NSFNET. This is because in a dense network, there are many alternative paths with lengths close to or equal to the length of the shortest path. Therefore, rerouting a lightpath to an alternative path does not reduce the overall resource efficiency. However, in a sparse network, the alternative paths may be far longer than the shortest path. Rerouting a lightpath to a longer alternative path may reduce the overall resource efficiency, which in turn may compromise the effect of rerouting.

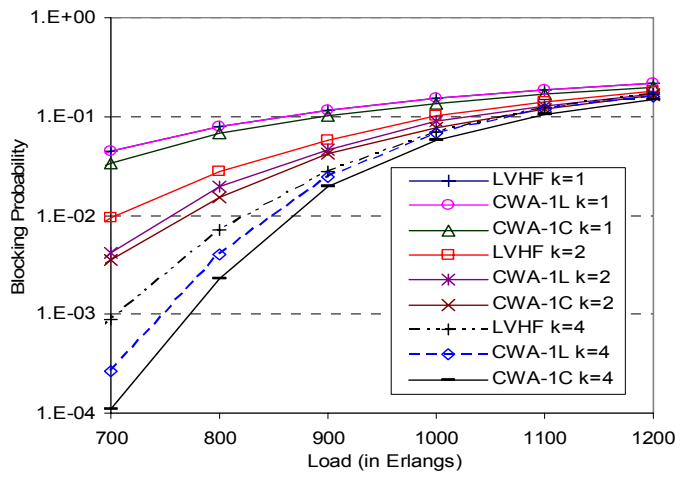

Fig. 5. Simulation results for the NSF network. $k$ (the number of alternate paths) is 1,2 and 4 respectively.

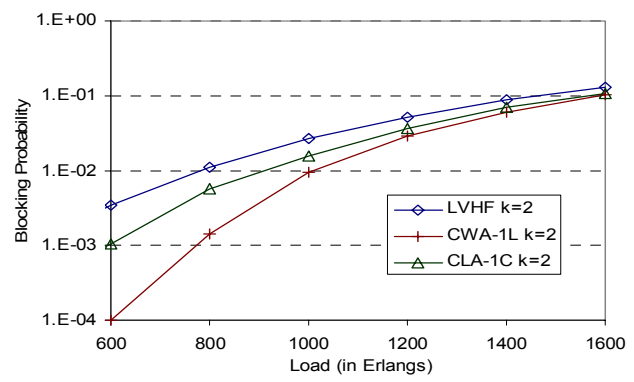

(a)

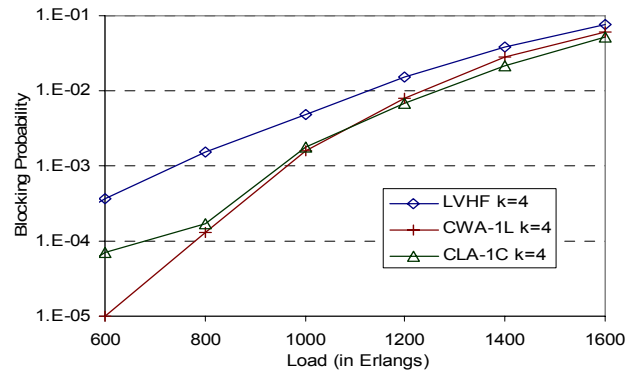

(b)

Fig. 6. Simulation results for the EUPAN network. (a) $k$ (the number of alternate paths) $=2$, (b) $k=4$.

\section{REFERENCES}

[1] J. Anderson, J. S. Manchester, A. R. Moral, M. Veeraraghavan, "Protocols and Architectures for IP Optical Networking," Bell Labs Technical Journal, January 1999.

[2] A. Lardles, R. Gupta, R. A. Patterson, "Traffic grooming in a multi-layer network," Optical Networks Magazine, May 2001.

[3] K. Zhu, B. Mukherjee, "On-Line Approaches for Provisioning Connections of Different Bandwidth Granularities in WDM Mesh Networks," OFC 2002, pp.549-551, March 2002.

[4] K. Lee, V. O. K .Li, "A wavelength rerouting algorithm in wide-area alloptical networks," Journal of lightwave technology, vol. 14, no. 6, June 1996.

[5] G. Mohan, C. Siva Ram Murthy, "A time optimal wavelength rerouting algorithm for dynamic traffic in WDM networks," Journal of lightwave technology, vol. 17, no. 3, March 1999.

[6] E. Mannie, "Generalized Multi-Protocol Label Switching (GMPLS) Architecture," Internet draft, draft-ietf-ccamp-gmpls-architecture-04.txt, February 2003, work in progress.

[7] "Dynamic Traffic Grooming using Fixed-Alternate Routing in WDM Mesh Optical Networks", to appear in the Workshop on Traffic Grooming 2004. 